\title{
Velocity of detonation (VOD) measurement techniques practical approach
}

\author{
Aruna D.Tete ${ }^{1}$, Dr. A.Y.Deshmukh², Dr. R.R.Yerpude ${ }^{3}$ \\ ${ }^{1}$ Department of Electronics Engineering, G.H.Raisoni College of Engineering, Nagpur \\ ${ }^{2}$ Department of Electronics Engineering, G.H.Raisoni College of Engineering, Nagpur \\ ${ }^{3}$ Department of Mining Engineering, Visvesvaraya National Institute of Technology, Nagpur \\ *Corresponding author E-mail: aruna.mathurkar@gmail.com
}

\begin{abstract}
Velocity of Detonation (VOD) is an important measure characteristics parameter of explosive material. The performance of explosive invariably depends on the velocity of detonation. The power/ strength of explosive to cause fragmentation of the solid structure determine the efficiency of the Blast performed. It is an established fact that measuring velocity of detonation gives a good indication of the strength and hence the performance of the explosive. In this survey various VOD measurement techniques such as electric, nonelectric and fibre optic have been discussed. To aid the discussion some commercially available VOD meter comparison are also presented. After review of the existing units available commercially and study of their respective merits and demerits, feature of an ideal system is proposed.
\end{abstract}

Keywords: Velocity of Detonation (VOD), Confined, Shock wave, Slurry, Blast hole

\section{Introduction}

Explosives are used extensively for mining and other civil use like tunnel, well making, building demolition etc. The performance of explosive invariably depends on the Velocity Of Detonation. The rate at which the detonation wave travels through an explosive column is called the Velocity of Detonation (VOD). It is one of the most important property of explosive on which its performance is dependent. It is essential to use explosives in a optimum quantity for better results, avoiding wastage at the same time for maximizing the performance.

It is an established fact that measuring velocity of detonation gives good indication of the strength and hence the performance of explosive. This VOD value could be typically from $2500 \mathrm{~m} / \mathrm{s}$ to upto $7000 \mathrm{~m} / \mathrm{s}$ depending on the type and form of the explosive used. The typical lengths of explosives involved are from $0.2 \mathrm{~m}$ to several $10 \mathrm{~s}$ of meters. Correspondingly the time span of the measurement could be from 50useconds to few 10s of milliseconds. Thus it is essentially a very short duration phenomenon.

Usually these VOD values are based on laboratory measurements and empirical calculations and hence theoretical values. Evaluation of the blast design is carried out with the assumption that the explosives have performed as per the specification, which may not be true in all cases. A reduction in the VOD will cause a reduction in the detonation pressure as well as in the availability of the shock energy of the explosive. Beside all these degree of fragmentation, ground vibration produced and safety in charging and handling of explosives are equally important parameters. It is important to establish the relationship between the product's VOD characteristics to the rock environment, blast design and other measurements. The selection and evaluation of the explosive performance depends on the properties of explosive used which directly affect the blast performance. The important properties of explosive are density, velocity of detonation, detonation pressure, sensitivity, energy strength, chemical stability, water resistance, thermal stability and fume characteristics.

Experimentation with the existing blast conditions have shown that all explosives deteriorate progressively in wet holes. Since the concentration has been made on the in-the-hole measurement of VOD, it is observed that there are many parameters which affects the velocity of detonation like confinement, formulation characteristics, density, sensitizing agents, temperature and temperature cycling, primer size and type, sleep time in blast hole, borehole loading techniques, blast design, explosive column length, blast environment, storage-shelf life and mixing in bulk loading system. 
Worldwide some VOD meters are available to measure velocity of detonation. VODMate from Instantel and Minitrap and Handitrap from MREL are based on resistance wire continuous VOD system, some of them can also use probe rod. In this method constant current is passed through a resistive wire pair which gets shortened continuously due to detonation. The rate of voltage drop gives VOD. SpeedVOD, ShotTrack are system which are based on Time Domain Reflectometer (TDR). The unit sends an electrical pulse down a coaxial cable and measures the time for the pulse to be reflected from the end of the cable. VODEX-100A uses point to point technique. It relies on the highly charged plasma generated within the explosion detonation front to sequentially increase the electrical conductivity at the end of series of wire pairs located at desired positions in the charge. Timers associated with these points directly gives VOD. Whereas FO-2000P uses point to point fiber optic system for VOD measurement. Cost of consumable for such system is very high. The resistive technique's resolution advantage was compromised by noise, whereas ShotTrack has a limitation of incorrect readings when joiner is used.

Presently various types of explosives are manufactured in India by more than 25 companies under different trade names. However most of these manufacturers rely on laboratory measurement for specifying VOD values. These are based on chemical composition of explosive and some empirical calculations. Some of them do carry out VOD measurement on cartridge form in unconfined space. It is apparent that such approach may not give an accurate and authentic representation of VOD value of an explosive, in-hole. In the evaluation study ${ }^{[3]}$ it is observed that out of the 76 blasts carried out for

VOD measurement only 56 was successful recording. It listed out various reasons for no recording in 20 cases. Some of the major reasons for failure of recording were requirement of skilled man power and very careful setup and planning for VOD measurement. Some of the factors for local manufacturer's reluctance to use such sophisticated VOD measuring instruments are

- $\quad$ requirement of skilled manpower for conducting a test,

- $\quad$ difficult to setup the measurement system in foolproof way within the timeframe available,

- $\quad$ high cost of measurement system and consumables,

- $\quad$ recording obtained still needs to be analyzed while rejecting or filtering out noise data,

- $\quad$ some amount of discretion is involved before presenting the record data or values,

- $\quad$ non existence of strict rules and regulations for maintaining VOD of explosive,

- $\quad$ no penalization involved if VOD values are not within acceptable range

In fact in most cases measurement of in-hole VOD does not even form part of any regular or periodic testing procedure. This is also because no reliable and practical instrument is available to do the measurement quickly.

Some of the constraints observed in use of present day equipment available for VOD measurement are non pickup of proper start signal, cutoff of connecting cable by fly-rock, electrical noise generated during explosion, non uniformity in interconnecting cable (in case instrument is based on Corrtex method), requirement of expensive and complex software to device VOD value, result are still dependent on user interpretation of record data.

In this paper a design approach towards a VOD measurement unit is discussed which includes continuous wire discrete resistance type VOD measurement technique. In this technique step change in resistance is preferred to increase the system reliability. System allows joiner if required and noise interference is minimized. Data acquisition unit is capable of direct display of VOD.

\section{VOD measuring techniques}

Detonation velocity is an important property to consider when rating an explosive. It has been observed that most of the manufacturers and the utilities rely on calculations based the chemical composition of the bulk explosive to arrive at the VOD value. Needless to mention such interpretation can be fairly inaccurate due to variations in raw material quality, manufacturing process followed and more importantly it is still a theoretical value only. Deriving the correct value of VOD with the proper measurement techniques will result in reduction in the consumption of explosives with optimized results. Presently the explosives used can be in two forms Cartridge \& Bulk. Cartridge is a packed form in tubular shape while bulk is free flowing slurry which is put in the hole from a pump truck. Detonation velocity may be expressed as a confined or unconfined value and is normally given in meter per second $(\mathrm{m} / \mathrm{s})$. The confined detonation velocity measures the speed at which the detonation wave travels through a column of explosive within a borehole or other confined space. The unconfined velocity indicates this rate when the explosive is detonated in the open. Explosives generally are used under some degree of confinement. 


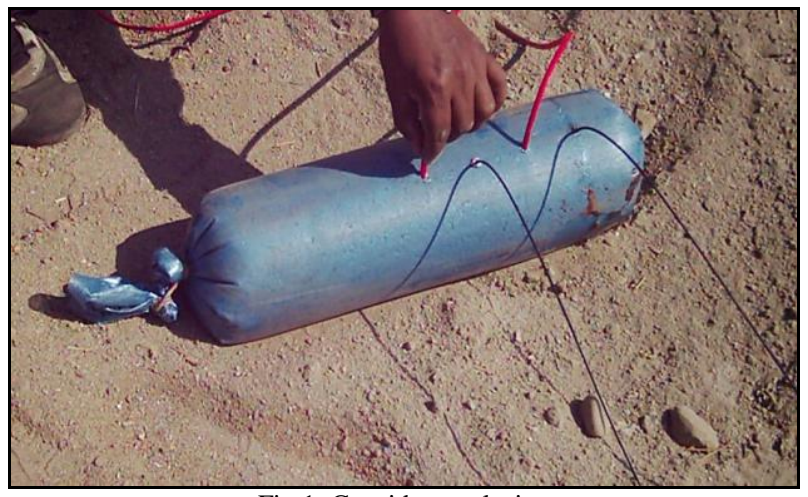

Fig.1: Cartridge explosive

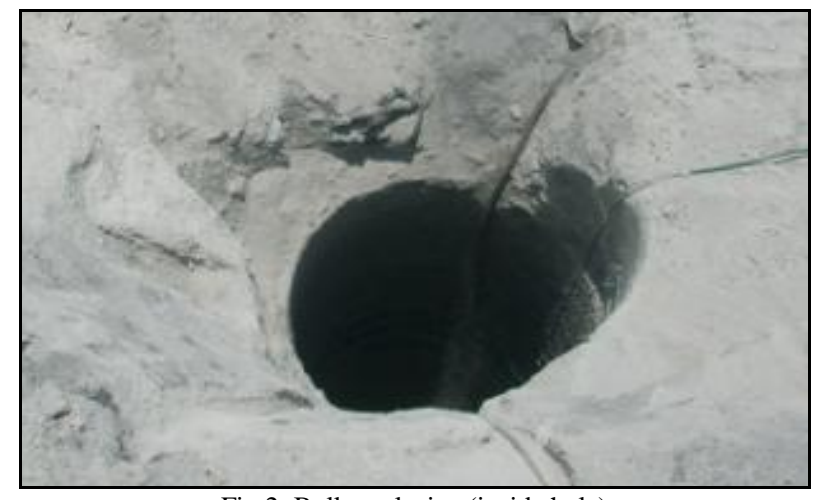

Fig.2: Bulk explosive (inside hole)

There are number of VOD measuring techniques are available worldwide, each possessing it's advantages and disadvantages. In this paper various techniques are discussed, comparative study is given and attempt has been made to find a cost effective, easy to operate, reliable and accurate method of measuring VOD of bulk explosive in a hole. Based on the method adapted an instrument would be designed and tested.

Existing practices for measurement of VOD adapted worldwide can be broadly classified into methods listed as follows

a) Dautriche Method ${ }^{2}$ - In this method two detonation wave propagating from both the ends of explosive column via a detonating fuse bound on a aluminum plate collide. The distance of collision mark from mid point is measured. This distance is directly proportional to Velocity of Detonation. This method of VOD measurement is suitable for unconfined space where the explosives are used in cartridge form.

b) Photographic Method - Another method in the category is photographic method, where detonation wave is monitored continuously using Streak and framing (high speed) camera. Explosion is an auto luminous process. The light emitted is captured continuously in real time. The VOD can be easily calculated from the motion video.

c) Discrete points (Point to point) Electric Method - Point to point VOD systems are basically supported by electronics start and stop timer. The one end of sensor cables are inserted into the explosive column at varying distance and other end to the VOD recorder where the start and stop signals are recorded. When detonation reaches the first sensor timing clock is started and the following sensor cable send the stop signals when detonation reaches to it. The distance between sensor cables are known and thus VOD can be calculated. This method is limited in providing information for critical experiments because of discontinuity of the sensor cable in the explosive column.

d)Resistance Wire Continuous VOD method - This method was developed in early 1960s by the United States of Mines(USBM) This method basically follow the Ohm's Law(E=RI) where E=Voltage, R=Resistance and I=Current. In this method ionization caused by explosion provides electric short continuously causing the voltage drop monitored by the instrument which is equivalent to the change in resistance value and the constant current. Thus a voltage drop can be measured instantaneously at any point in time. In this method single wire or two twisted wire of known resistance can be acted as single or double sensors respectively at the same time. It is observed in the experimentation that if in this method sensors are not ruggedized or distance with the return path is not maintained properly for shorting during detonation then the results are usually undeciphered or no readings are obtained.

e) SLIFER continuous VOD system - The SLIFER (Shorted Location Indication by Frequency of Electrical Resonance) system was originally developed by Sandia National laboratories to measure the propagation of shock waves from nuclear explosion. It consist of a shorted length of coaxial cable as a sensor in the explosive column which is connected to the oscillator circuit. This small device show the frequency which is controlled by length of sensor in the explosive column. As the wire length decreases frequency of oscillation increases. By monitoring this frequency as a function of time, the rate of cable length change can be determined, leading directly the measurement of VOD. This system has been limited to laboratory work. The restriction with the SLIFER system is that recording cable length with the oscillator is $66 \mathrm{~m}$ per channel, moreover each sensor must have oscillator connected in a line which should be placed close to the hole or shot area.

f) TDR continuous VOD system - The TDR system originally developed by the Los Alamos National Laboratory to test and verify nuclear reaction yields and stress velocities into the surrounding medium. This system can also be called as CORRTEX system later changed to the VODR-I when the system was declassified for commercialization. In this system narrow electric pulse is sent through the cable sensor and return or reflected path is detected which is from the 
other end of sensor. This method does not require the sensing cable to be shorted in order to acquire date. This is one of the safest to use with any commercial or military explosive.

g) Method based on fiber-optic - In this method optical fiber is used which is capable of detecting and transmitting a light signal accompanying the detonation wave front. This method is point to point to type wherein the first cable signals the start whereas the second cable placed at a known fixed distance stops the timing clock. The fixed distance between probes divided by the timed clock directly gives the VOD value.

Based on the measuring techniques mentioned above various instruments are available worldwide and a brief comparison of the models available is given below.

Table 1: The analysis of the Models are provided in the following table

\begin{tabular}{|c|c|c|c|c|c|c|}
\hline Models $\Longrightarrow$ & \multirow[t]{2}{*}{ VODMate } & \multirow[t]{2}{*}{ Datatrap/Minitrap } & \multirow{2}{*}{$\begin{array}{l}\text { VODEX- } \\
100 \mathrm{~A}\end{array}$} & \multirow[t]{2}{*}{ Speed-VOD } & \multirow[t]{2}{*}{ Shot-Track } & \multirow[t]{2}{*}{ FO-2000P } \\
\hline Parameters & & & & & & \\
\hline $\begin{array}{l}\text { VOD measuring } \\
\text { technique }\end{array}$ & $\begin{array}{l}\text { Continuous } \\
\text { Wire, } \\
\text { Electrical }\end{array}$ & $\begin{array}{l}\text { Continuous Wire, } \\
\text { Electrical }\end{array}$ & $\begin{array}{l}\text { Discrete } \\
\text { Points Wire, } \\
\text { Electrical }\end{array}$ & TDR & TDR & Fiber optic \\
\hline Resolution & $<10 \mathrm{~mm}$ & $<10 \mathrm{~mm}$ & $<5 \mathrm{~mm}$ & $62 \mathrm{~mm}$ & $62 \mathrm{~mm}$ & $<5 \mathrm{~mm}$ \\
\hline Noise influence & Susceptible & Susceptible & Susceptible & Immune & Immune & Immune \\
\hline $\begin{array}{l}\text { Set-uptime } \\
\text { requirement }\end{array}$ & $5-15$ min. & $10-20 \mathrm{~min}$. & $20-30 \mathrm{~min}$. & $10-20$ min. & $10-20 \mathrm{~min}$ & $\begin{array}{l}10-20 \\
\text { min. }\end{array}$ \\
\hline Consumable required & Sensor cable & $\begin{array}{l}\text { Probe rod or } \\
\text { cable }\end{array}$ & $\begin{array}{l}\text { Ribbon } \\
\text { cable }\end{array}$ & $\begin{array}{l}\text { RG-6, RG- } \\
58, \text { RG-59 }\end{array}$ & $\begin{array}{l}\text { RG-6, RG- } \\
58, \text { RG-59 }\end{array}$ & $\begin{array}{l}\text { Fiber optic } \\
\text { cable }\end{array}$ \\
\hline $\begin{array}{l}\text { Type of manpower } \\
\text { required for set-up }\end{array}$ & Skilled & Skilled & Semi-skilled & Skilled & Skilled & $\begin{array}{l}\text { Semi- } \\
\text { skilled }\end{array}$ \\
\hline Cost of consumable & Low & High & Average & Moderate & Moderate & Very High \\
\hline Multihole capability & Yes & Yes & Yes & Yes & Yes & Yes \\
\hline $\begin{array}{l}\text { Continuous } \\
\text { measurement } \\
\text { capability }\end{array}$ & Yes & Yes & No & Yes & Yes & No \\
\hline Software capability & Yes & Yes & Yes & Yes & Yes & Yes \\
\hline $\begin{array}{l}\text { Direct display of } \\
\text { VOD }\end{array}$ & No & No & Yes & No & No & Yes \\
\hline $\begin{array}{l}\text { Suitability for } \\
\text { waterlogged holes }\end{array}$ & Not known & Not known & Not known & Yes & Yes & Yes \\
\hline
\end{tabular}

\section{Typical field observations}

Typical observations of available VOD meters with their recorded data of blast are shown in the Fig. 3 to Fig. 8 . Fig. 3 and 4 show the records obtained from ShotTrack and Minitrap respectively. The blast employed one meter length of heavy ANFO in 150mm PVC tubing (Short charge length). It was seen that the Minitrap unit was completely unsuccessful in recording the VOD over any part of the charge length whereas ShotTrack yielded a VOD of $3.2 \mathrm{Km} / \mathrm{s}$ over $40 \mathrm{~cm}$ of trace. VOD result obtained by Instantel's VODMate (Fig.5) for $7 \mathrm{~m}$ of SMS (Site Mix System) explosive column using $8.234 \Omega / \mathrm{m}$ probe cable. Some noise spikes are observed in the initial stages. As in Fig. 6 the snapshot of VODSYS-4 are shown where $4.4 \mathrm{~m}$ of explosive column is used. Trial with Multihole blasting was performed using ShotTrack VOD meter, it was observed (Fig.7) that records were achieved using a joiner to connect the in-hole coaxial cable leading away to the ShotTrack unit. The use of joiner in this work represents the worst case for taking reflectometer measurement because reflection off the joiner may provide a stronger reflection than off the end of the cable. In such cases, cable past the joiner would not be monitored by the unit. The unit recorded reflection off the joiner than the VOD. It is seen that almost all of the unit require data to be transferred, properly selected and analyzed for VOD figure calculation. 
Below table gives a brief on work done by NIRM [3] using various instruments available and results obtained.

\begin{tabular}{|c|c|c|c|c|}
\hline Mine & Instrument & Condition & $\begin{array}{l}\text { No.of Blasts } \\
\text { Recorded }\end{array}$ & $\begin{array}{l}\text { No.of events } \\
\text { availble }\end{array}$ \\
\hline GDK OCP-1 & VODSYS -4,MREL & \multirow{5}{*}{$\begin{array}{l}\text { Confined } \\
\text { (in-the-hole) }\end{array}$} & 33 & 9 \\
\hline $\begin{array}{l}\text { Walayar Limestone mine, } \\
\text { ACC }\end{array}$ & VODMATE, Instantel & & 5 & 3 \\
\hline $\begin{array}{l}\text { Jayantipuram Limestone } \\
\text { Mine,MCL }\end{array}$ & VODMATE, Instantel & & 6 & 9 \\
\hline GDK OCP-1 And -3 & Micro Trap, MREL & & 18 & 17 \\
\hline GDK OCP-1 And -3 & VODMATE, Instantel & & 7 & 11 \\
\hline \multirow[t]{2}{*}{ GDK OCP-1 } & $\begin{array}{lll}\text { VODSYS } & -4, \text { MREL } & \text { and } \\
\text { Micro Trap, MREL } & \\
\end{array}$ & Unconfined(surface) & 7 & 7 \\
\hline & & Total & 76 & 56 \\
\hline
\end{tabular}
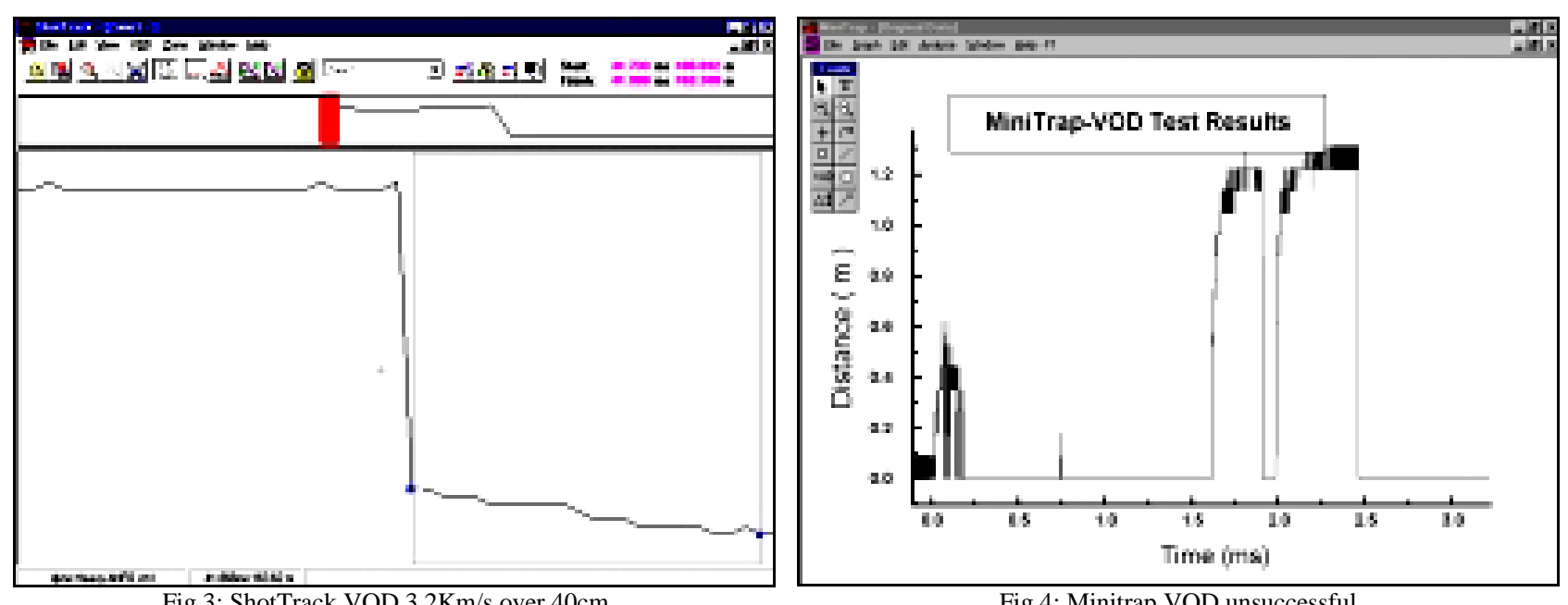

Fig.3: ShotTrack VOD $3.2 \mathrm{Km} / \mathrm{s}$ over $40 \mathrm{~cm}$

Fig.4: Minitrap VOD unsuccessful

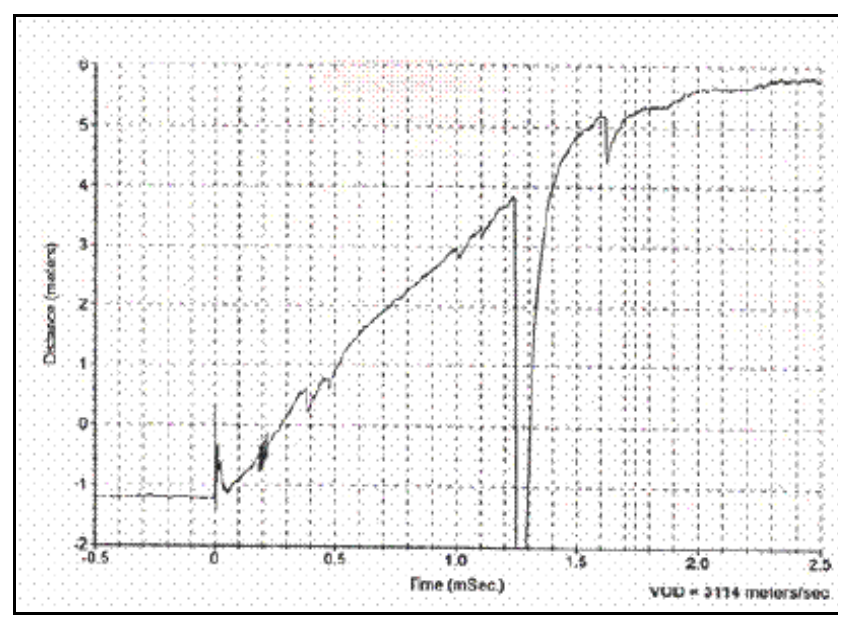

Fig.5: VOD result for Instantel VODMate

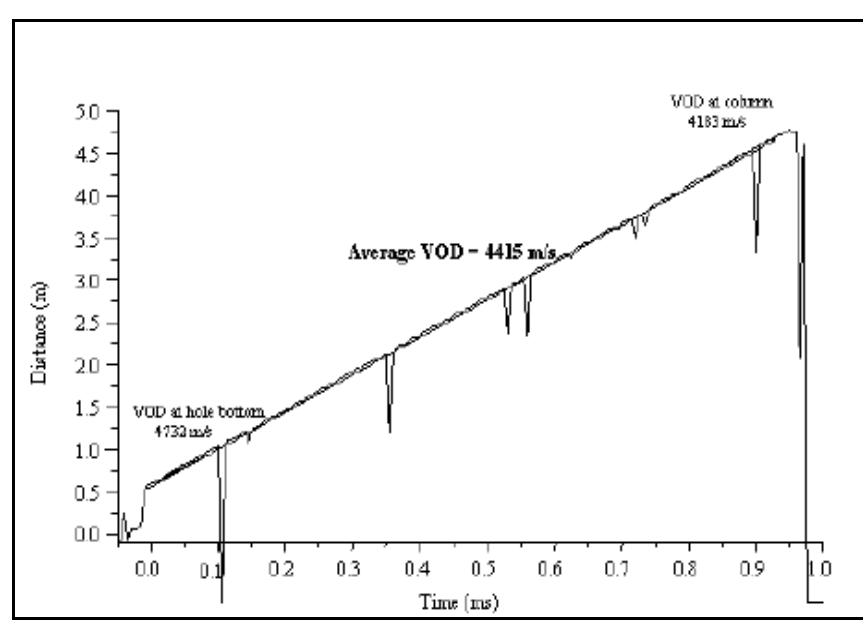

Fig.6: Full trace VOD by VODSYS 


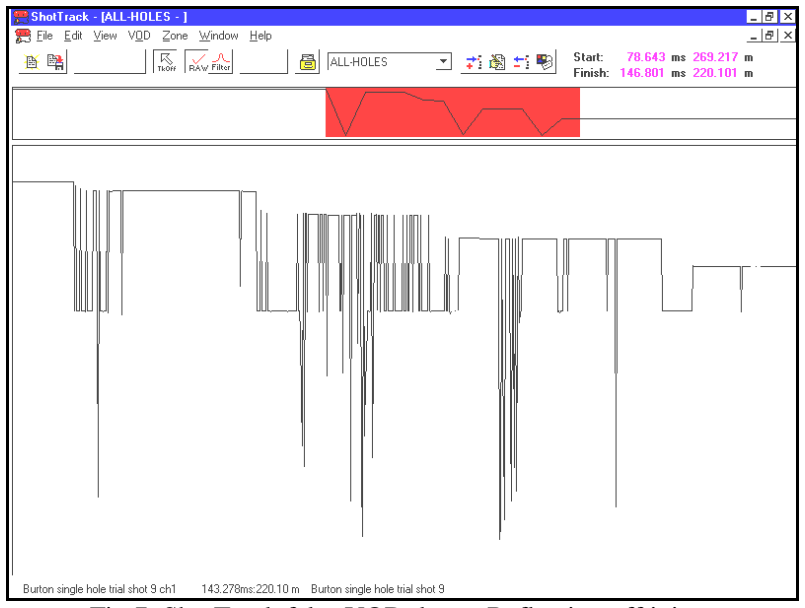

Fig.7: ShotTrack false VOD due to Reflection off joiner

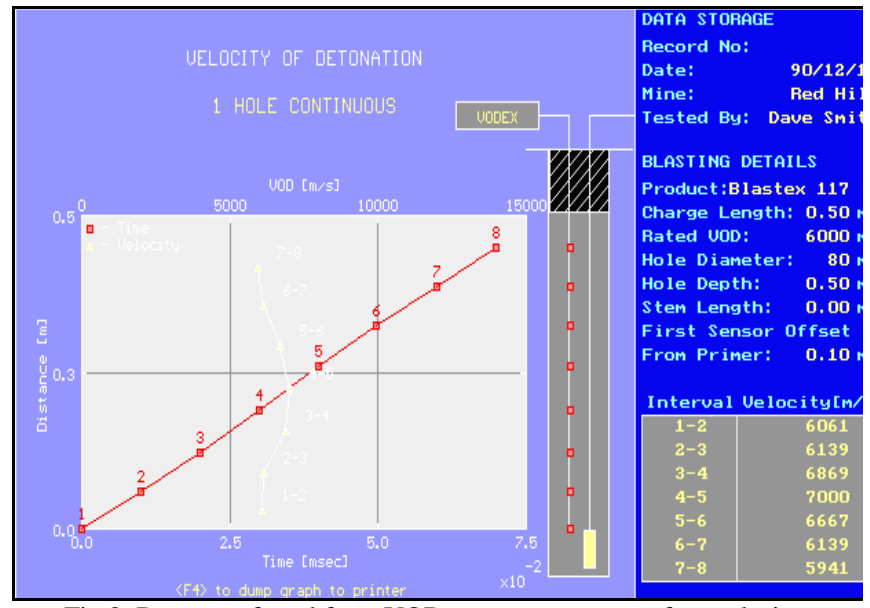

Fig.8: Data transferred from VOD meter to computer for analysis

\section{Discussion}

It may be seen from the above comparison table that most of the units are sophisticated and costly. They require skilled man power to operate it. Also very few have direct VOD display capability. Cost of consumables varies from moderate to very high, The main aim of this study is to arrive at a suitable method which is moderate in cost, with low cost of consumables, and one which does not require skilled man power and most importantly simply gives direct display of VOD value without requiring any software skills. Measurement of continuous value of VOD may not be required. Whereas most of the equipments though sophisticated and good for continuous VOD measurement are not suitable for routine VOD value measurement because of requirement of skilled man power and high cost of consumables.

It may be observed that continuous resistive wire is the least cumbersome method and easy for setup with low cost of consumable. However due to continuous monitoring huge amount of data is gathered which can be handled only by means of software and hence requires skilled manpower. Also considerable time and skill is required to select appropriate data thus making it a rather time consuming and tedious process. A unit like VODEX-100A which gives direct display of VOD requires careful and is not user friendly. Also accuracy level is not as good as continuous wire method. A supervising microprocessor circuit controls the counting operation and calculates VODs, stores recorded data and provides the means to communicate with DOS or Windows compatible computers. Optional PC compatible software allows data from the VODEX-100A to be transferred to a PC for analysis, graphical presentation and permanent storage. Fiberoptic method which is not susceptible to noise is a good method but requires costly consumables.

The evaluation of ShotTrack_VOD system and MREL Minitrap system has been done ${ }^{[10]}$. ShotTrack uses Time domain Reflectometer technique and Minitrap uses continuous wire resistive method. After the evaluation it has been seen that The MREL Minitrap system performed poorly when tested against ShotTrack in field tests. As a resistive type VOD system, Minitrap was potentially capable of superior operation on small charge lengths due to its finer resolution. The resistive technique's resolution advantage is however severely compromised by its main weakness - which is noise. It is also seen in ShotTrack VOD system that joiner in the cable should be avoided in TDR technique which may give the false VOD due to reflection off joiner.

This paper offers a view towards design of an unit which has a low cost sensor, is easy to install/ setup, reduced susceptibility to electrical noise, design sufficient enough to be able to calculate and display VOD directly without compromising accuracy. An ideal sensor should have large change in resistance for the given blast length. Sensor should be based on change in resistance either continuous or in steps. Step change in resistance is preferred as it reduces the system complexity as well as increase reliability. System allow joiner if required. Use of only a particular grade of cable should not be a prerequisite i.e. system should be able to accommodate at least a certain range of cable/wire types. Sensor system should be flexible enough to be suitable for explosive length from $10 \mathrm{~cm}$ to $10 \mathrm{mtrs}$ moreover it should be easy to setup and connect. Sensor and it's acquisition unit should have self diagnostic feature to detect sensor fault. As noise is generated during explosion, so sensor and it's acquisition system should minimize noise interference. Sensor should not be affected by presence of moisture or other conducting media. Design should allow sufficient interconnecting cable length so that unit can be kept at a safe distance from explosion, since some portion of cable gets destroyed in explosion so cable should not be very costly. System should not require elaborate manual interpretation of data acquired to calculate VOD. Design should be optimized such that it is able to give only VOD output for given requirement of in hole VOD. Nothing more nothing less continuous VOD though preferable is not desirable as it causes 
much complexity and is difficult to interpret, rather continuous wire discrete resistance is less complex, reliable and easy to interpret.

\section{Conclusion}

The sensor used for continuous wire method is a high resistance core wire with shielding. However the resistance per meter is still very low about few couple of ohms which makes it susceptible to noise and constrained by total wire length. Resistance change of more than $0.4 \%$ is required. Sensor used in discrete points method is based on resistance change in the region of several kilo ohms improving the noise immunity, however has drawback non continuous VOD measurement as well as difficult to setup. Instruments like Speed VOD and Fiber optic use costly cables as sensor. A sensor with appreciable change in resistance per meter could be ideal. Also instead of continuous monitoring if the changes are in steps large data storage and analysis capability will not be required. Thus a sensor which can provide change in resistance in steps could be ideal. This sensor has to ensure proper change in resistance under the given circumstances faithfully.

\section{References}

[1] Edwin M.Chan, Vivian Lee, Samuel P. Mickan, Phil J. Davies "Low cost optoelectronics devices to measure velocity of detonation", Proceedings of SPIE Vol. 5649(SPIE, Bellingham, WA,2005)

[2] M. Pradhan, "Effect of Charge Temperature on the detonation Velocity of Bulk Emulsion Explosives" IE(I) Journal-MN vol.90, August 2009.

[3] "Evaluation of explosive performance through in-the-hole detonation velocity measurement" by National Institute of Rock Mechanics August 2001.

[4] J.Benterou, E. udd, P.Wilkins, F.Roeske, E.Roos, D.Jackson "In-Situ Continuous Detonation Velocity Measurements using Fiber-optic Bragg Grating Sensors"

[5] Europhyro 2007, 34th International Pyrotechnics Seminar Beaune, France October 8, 2007 through October 11,2007

[6] Lun K. Cheng and Jan van Bree "Fibre-optic sensors in explosion and detonation experiments" SPIE 10.1117/2.1200705.0729

[7] Jerry.Benterou, Corey V. Bennett, Garrett Cole, D.E. Hare, Chadd May, Eric Udd "Internal Detonation Velocity Measurement Inside High Explosives", SPIE Defence Security and Sensing Orlando, FL, United States April 13, 2009

[8] M.J.Louw, R.S.Sarracino and S.M.Vather, "Comparison of the theoretical and measured velocities of detonation for selected eexplosives" Journal of The South African Institute of mining and Metallurgy.vol.93, no.6.June 199300 147-153

[9] Smart structure devices and system II, proceeding of SPIE vol.5649 (SPIE, Belingham, WA2005)

[10] Gil Smith "ShotTrack VoD system evaluation", MATRIX Consultants Australia Pty Ltd June 2000. 